\title{
Pre-Extensively Drug Resistant Tuberculosis (Pre-XDR-TB) among Pulmonary Multidrug Resistant Tuberculosis (MDR-TB) Patients in Bangladesh
}

\author{
Tamanna Tasnim ${ }^{*}$, Shirin Tarafder1, Fatema Mohammad Alam1, Humayun Sattar ${ }^{1}$, \\ S. M. Mostofa Kamal ${ }^{2}$
}

${ }^{1}$ Department of Microbiology and Immunology, Bangabandhu Sheikh Mujib Medical University (BSMMU), Dhaka, Bangladesh ${ }^{2}$ National Tuberculosis Reference Laboratory (NTRL), National Institute of Diseases of the Chest and Hospital (NIDCH), Dhaka, Bangladesh

Email: *tamannatasnim02@yahoo.com

How to cite this paper: Tasnim, T., Tarafder, S., Alam, F.M., Sattar, H. and Mostofa Kamal, S.M. (2018) Pre-Extensively Drug Resistant Tuberculosis (Pre-XDR-TB) among Pulmonary Multidrug Resistant Tuberculosis (MDR-TB) Patients in Bangladesh. Journal of Tuberculosis Research, 6, 199-206.

https://doi.org/10.4236/itr.2018.63018

Received: June 25, 2018

Accepted: July 21, 2018

Published: July 24, 2018

Copyright $\odot 2018$ by authors and Scientific Research Publishing Inc. This work is licensed under the Creative Commons Attribution International License (CC BY 4.0).

http://creativecommons.org/licenses/by/4.0/

\begin{abstract}
Background \& Objectives: Emergence of drug resistant Tuberculosis (TB) is a major obstacle in the TB control programme of Bangladesh. This study was carried out to detect pre-extensively drug resistant TB (pre-XDR-TB) cases among the multidrug resistant TB (MDR-TB) patients in Bangladesh, as the early detection of pre-XDR-TB can guide clinicians in the appropriate modification of MDR-TB treatment regimen with effective drugs to prevent treatment failure. Methodology: A total of 68 MDR-TB cases were enrolled in this study. Multiplex Real-time PCR was done to detect pre-XDR-TB cases directly from sputum samples of MDR-TB patients. Results: Out of 68 MDR-TB cases $11(16.18 \%)$ cases were detected as pre-XDR-TB. The resistant profile of the 11 pre-XDR-TB revealed 9 (81.82\%) cases of fluoroquinolone (FLQ) resistant pre-XDR-TB and $2(18.18 \%)$ cases of injectable second line (ISL) agent resistant pre-XDR-TB. Out of 11 pre-XDR-TB cases 7 (63.64\%) cases had history of taking treatment for MDR-TB regularly, $1(9.09 \%)$ case had history of taking treatment for MDR-TB irregularly and $3(27.27 \%)$ cases had no history of taking treatment for MDR-TB. Conclusion: This study encountered a high rate of pre-XDR-TB cases along with a significant number of primarily resistant bacilli which is of concern in the management of MDR-TB. It is evident that Bangladesh is in urgent need to device strategies for rapid and early detection of pre-XDR-TB in order to prevent treatment failure of MDR-TB cases and also to halt the progression of MDR-TB cases to extensively drug resistant $\mathrm{TB}$ (XDR-TB), which is not only difficult but also very expensive to treat.
\end{abstract}




\section{Keywords}

Pre-XDR-TB, MDR-TB, Bangladesh

\section{Introduction}

Tuberculosis (TB) is a major public health problem in Bangladesh. The estimated incidence rates for all forms of TB in 2015 were 225 per 100,000. An estimated 45 per 100,000 died of TB in the same year. The prevalence of MDR-TB (Multi drug resistant TB) in Bangladesh in new cases is $1.6 \%$ and in re-treatment cases is $29 \%$ [1].

Pre-extensively drug resistant tuberculosis (pre-XDR-TB) is a comparatively new term and is defined as TB with resistance to rifampicin (RMP) and isoniazide (INH) with additional resistance to either a FLQ (Fluoroquinolone) or ISL (Injectable second line) agent but not against both these drugs simultaneously [2]. Thus pre-XDR-TB cases with FLQ or ISL resistance receive less number of effective drugs under standard MDR-TB regimen. It may amplify further resistance to the effective drugs and progression towards XDR-TB (Extensively drug resistant TB).

XDR-TB is defined as TB resistant to RMP and INH (MDR-TB) with additional resistance to second line anti-TB drugs i.e. to any FLQs, and to at least one of the three injectable second-line drugs (ISL) naming amikacin, kanamycine and capreomycin [3]. The prevalence of XDR-TB is $9.5 \%$ worldwide [4]. Treatment of XDR-TB is complicated, as it requires the use of second-line drugs that are less effective and more toxic, thus demanding longer treatment duration. Detection of pre-XDR-TB cases among MDR-TB patients is an important step in the prevention of treatment failure of MDR-TB and in addition, it helps to take appropriate measures to halt the progression towards XDR-TB.

So far no data is available regarding the status of pre-XDR-TB in Bangladesh. Neighboring countries like India, Pakistan and Nepal have reported high prevalence of pre-XDR-TB cases. In India 55.65\% pre-XDR-TB cases were found among pulmonary MDR-TB patients in a tertiary care hospital in Mumbai [3]. From Pakistan, two separate studies, one from Multan and the other from Karachi found the prevalence of pre-XDR-TB among MDR-TB patients to be $32.2 \%$ and $39.5 \%$ respectively [5] [6]. Nepal has reported $28 \%$ pre-XDR-TB cases among drug resistant Tuberculosis (DR-TB) cases [7]. Therefore the aim of our study was to detect the rate of pre-XDR-TB cases among pulmonary MDR-TB patients, admitted at the National Institute of Diseases of the Chest and Hospital (NIDCH), Dhaka, Bangladesh.

\section{Materials and Methods}

This cross sectional study was carried out from September 2016 to August 2017 at Bangabandhu Sheikh Mujib Medical University (BSMMU), Dhaka, Bangla- 
desh and the National Institute of Diseases of the Chest and Hospital (NIDCH), Dhaka, Bangladesh.

\subsection{Clinical Samples}

A total of 68 pulmonary MDR-TB patients (both newly diagnosed cases and patients under MDR-TB treatment regimen) attending the NIDCH were recruited in this study.

Inclusion criteria: Pulmonary MDR-TB patients diagnosed by Xpert MTB/RIF assay (Cepheid Inc, USA), which detected rifampicin resistance and by the conventional DST (drug susceptibility testing), which detected rifampicin and isoniazide resistance were selected.

Exclusion criteria: Extra pulmonary MDR-TB patients.

\subsection{Sample Collection and Processing}

Sputum samples were collected following standard protocol then digested and decontaminated by NALC-NaHO method [8].

1) Multiplex Real-time PCR: For the detection of pre-XDR-TB cases, Multiplex Real-time PCR was done on all sputum samples in the department of Microbiology of Bangabandhu Sheikh Mujib Medical University (BSMMU), Dhaka, Bangladesh. DNA extraction of the sputum samples was done using the Seegene DNA extraction kit (Seegene, Seoul, Korea) in accordance to the manufacturer's instruction. Real-time PCR was carried out on all extracted DNA samples using Anyplex II MTB/XDR detection kit (Seegene, Seoul, Korea), in Bio-Rad's 96 well PCR plate (Bio-Rad Laboratories, Hercules, CA, USA), in accordance to the manufacturer's instruction. The Anyplex II MTB/XDR kit identifies Mycobacterium Tuberculosis Complex (MTC) and mutations conferring resistance to FLQ ( gyrA) and ISL (rrs and eis promoter region). The kit detects $13 \mathrm{XDR}$ associated mutations (7 FLQ and 6 ISL resistance mutations) utilizing the $\mathrm{DPO}^{\mathrm{TM}}$ (dual priming oligonucleotide) and TOCE $^{\mathrm{TM}}$ (tagging oligonucleotide cleavage and extension) technologies for the simultaneous real-time detection of multiple point mutations with high specificity.

2) PCR Procedure: Each PCR solution consisted of $5 \mu \mathrm{L}$ of the extracted DNA added to $15 \mu \mathrm{L}$ of master mix $(4 \times$ TOCE oligo mix for XDR, $4 \times$ Anyplex PCR master mix, and RNase-free water). One positive control, one negative control and one wild type control were added in separate wells containing master mix during each PCR run. For positive control $5 \mu \mathrm{L}$ of MTB/DR PC, for negative control $5 \mu \mathrm{L}$ of RNase-free water and for the wild type control $5 \mu \mathrm{L}$ of MTB/DR WTC were added inseparate wells containing master mix, during each PCR run. The plate was sealed with plate cover and then spun in the centrifuge very briefly to mix the contents of the wells properly and to remove air bubbles. The blunt end of a sharpie was used to seal the gaps between the wells to prevent the evaporation of the contents of wells during the PCR process. The plate was loaded into the plate holder in the PCR machine and plate lay out was designed 
in the software. The PCR run method was then set in the machine. Amplification was performed using the CFX96 Real-Time System (Bio-Rad Laboratories, Hercules, CA, USA), and the melting-curve data analyses and interpretation were performed automatically with a connected computer, using Seegene viewer software, version 2.0 (Seegene Technologies, Concord, CA, USA).

3) PCR result Interpretation: MDR-TB: Simultaneous detection of any of the $7 \mathrm{INH}$ mutations (4 kat $G$ gene mutations, $3 \mathrm{inh} A$ promoter mutations) and any of the 18 RMP mutations ( $r p o B$ gene) were classified as MDR-TB. Pre-XDR-TB: Detection of INH and RMP mutations, along with either the FLQ (4 gyr $A$ gene mutations) or the ISL (3 rrs and eis promoter region) mutations, but not both types of genes simultaneously, were classified as pre-XDR-TB. Pre-XDR-TB (FLQ): Detection of INH and RMP mutations along with FLQ ( $g y r A$ gene) mutation. Pre-XDR-TB (ISL): Detection of INH and RMP mutations along with ISL (rrs and eis promoter region) mutation. XDR-TB: Detection of INH and RMP mutations, along with both the FLQ (4 gyrA gene mutations) and the ISL ( 3 rrs and eis promoter region) mutations simultaneously, were classified as XDR-TB.

\section{Results}

Out of the 68 MDR-TB patients, 11 (16.18\%) were detected as pre-XDR-TB cases. Table 1 shows the PCR results of the study population. Among the 68 MDR-TB patients, 14 (20.59\%) cases were PCR negative, 39 (57.35\%) cases were detected as only MDR-TB and 11 cases were detected as pre-XDR-TB, of which 9 (13.24\%) were FLQ resistant pre-XDR-TB cases, 2 (2.94\%) were ISL resistant pre-XDR-TB cases and the remaining 4 (5.88\%) were XDR-TB cases.

Age-wise distribution of pre-XDR-TB cases shows highest number of cases were in the age group of $21-30$ years followed by 3 cases in the age group of $\leq 20$ years. The 11 pre-XDR-TB cases were consisted of 7 males and 4 females cases (Table 2).

Table 3 shows the treatment status of pre-XDR-TB patients. Out of the 11 pre-XDR-TB patients, 7 (63.64\%) were taking standard MDR-TB regimen

Table 1. Results of Multiplex Real-time PCR in sputum samples of MDR-TB patients (n $=68)$.

\begin{tabular}{ccc}
\hline PCR & Number of patients (n) & Percentage (\%) \\
\hline PCR negative ${ }^{\mathrm{a}}$ & 14 & 20.59 \\
MDR-TB & 39 & 57.35 \\
Pre-XDR-TB & 11 & 16.18 \\
Pre-XDR-TB (FLQ) $+_{\text {Pre-XDR-TB (ISL) }{ }^{\mathrm{b}}}$ & $(09+02)$ & $(13.24+2.94)$ \\
XDR-TB & 04 & 5.88 \\
Total & $\mathbf{6 8}$ & $\mathbf{1 0 0}$
\end{tabular}

Note: a. PCR negative: No organism detected, no resistance detected; b. Pre-XDR-TB values in the table are the sum of pre-XDR-TB (FLQ) + pre-XDR-TB (ISL) values. 
Table 2. Baseline characteristics of the pre-XDR-TB cases $(n=11)$.

\begin{tabular}{cc}
\hline Characteristics & Number of patients (\%) \\
\hline Age & $3(27.27)$ \\
$\leq 20 \mathrm{yrs}$ & $5(45.45)$ \\
$21-30 \mathrm{yrs}$ & $2(18.18)$ \\
$31-40 \mathrm{yrs}$ & $1(9.10)$ \\
$41-50 \mathrm{yrs}$ & $0(00)$ \\
$>50 \mathrm{yrs}$ & \\
Sex & $7(63.64)$ \\
Male & $4(36.36)$ \\
Female &
\end{tabular}

Table 3. Treatment status of the pre-XDR-TB patients $(n=11)$.

\begin{tabular}{cc}
\hline Treatment status & Number of patients (\%) \\
\hline Taking standard MDR-TB regimen regularly & $7(63.64)$ \\
Taking standard MDR-TB regimen irregularly & $1(9.09)$ \\
Not taking standard MDR-TB regimen & $3(27.27)$ \\
\hline
\end{tabular}

regularly. There was only 1 (9.09\%) patient under standard MDR-TB regimen but had a history of taking the drugs irregularly and the remaining 3 (27.27\%) patients had no history of taking standard MDR-TB regimen.

\section{Discussion}

This study was conducted to observe the rate of pre-XDR-TB cases among MDR-TB patients in Bangladesh. Out of 68 MDR-TB patients, 11 (16.18\%) cases were found to have pre-XDR-TB. The findings of this study are similar to studies from India, South Africa and China where the detection of pre-XDR-TB among MDR-TB patients were $11.59 \%$ [9], 14.00\% [2] and 18.90\% [10] respectively. On the other hand several other studies have found much higher rates of pre-XDR-TB among their MDR-TB patients. In Nepal, a surveillance conducted by National TB Center in collaboration with WHO found the prevalence of pre-XDR-TB was $28 \%$ among registered DR-TB cases [7]. From China, Yuan et al. (2012) conducted a study by molecular characterization of the MDR-TB bacilli and observed 33.77\% pre-XDR-TB cases among MDR-TB patients [11]. From India two different studies have found the pre-XDR-TB cases among MDR-TB patients to be very high at $49.40 \%$ [12] and 55.65\% [3] respectively. It is important to note that these 2 studies were conducted in tertiary care private hospitals where patients are admitted with additional co-morbidities and with prior exposure to antibiotics like FLQs and aminoglycosides, which may be the reason for the higher resistance. From the aforementioned findings of the different studies, it is evident that the prevalence of pre-XDR-TB cases among the 
MDR-TB patients varies between countries and is some cases variations occur within the same county in different regions. These differences may be the result of different anti-TB regimens adopted by the different countries. Other contributing factors may be due to low socio-economic condition, poor health infrastructure and lack of sufficient medications in those regions.

The current study also detected higher number of FLQ resistant pre-XDR-TB (13.24\%) cases, than ISL resistant pre-XDR-TB (2.94\%) cases. Data from previous studies suggests that the incidence of FLQ resistant MDR-TB (pre-XDR-TB) is increasing worldwide [12]. The reason for the higher number of FLQ resistant pre-XDR-TB cases found in this study maybe due to the fact that FLQs are used indiscriminately in most of the common infections, including pneumonia and pyrexia of unknown origin, in addition to its use in MTB infection in Bangladesh. When FLQs are used as antibiotics they have two detrimental effects, first they have anti mycobacterial action which can delay the diagnosis of TB. Secondly, when these antibiotics are previously used for treatment, they can lead to selection of FLQ resistant MTB mutants. The exposures to FLQ antibiotics are more common than ISLs, as FLQ antibiotics are oral drugs, which are readily available in pharmacies without prescription in Bangladesh. ISLs are aminoglycosides (amikacin, kanamycin and capreomycin) which are injectable antibiotics and are also available without prescription in Bangladesh and they are also used for the treatment of bacterial infection other than TB. The indiscriminate use of these antibiotics may have contributed to the evolution of ISL resistant pre-XDR-TB cases found in this study.

The current study found a higher number of pre-XDR-TB cases in the age group of 21 - 30 years. Similarly two separate studies, one from India and the other from Nigeria also found higher numbers of pre-XDR cases among the young adult group, with ages ranging from 18 - 25 years and 15 - 29 years respectively [3] [13]. The probable cause of the higher numbers of drug resistant $\mathrm{TB}$ in the active age group may be due to their frequent movement, greater exposure to the environment, coming in contact with more people outdoors and higher case notification due to greater health awareness and concern among young adults.

The treatment status of the 11 pre-XDR-TB cases were also observed in this study, of which 7 (63.64\%) cases had history of taking MDR-TB drugs regularly, $1(9.09 \%)$ case had history of irregular MDR-TB treatment and the remaining 3 (27.27\%) cases were not under standard MDR-TB regimen. The high prevalence (63.64\%) of pre-XDR-TB cases under regular MDR-TB treatment regimen might be of consequence and alarming due to the higher transmission rate of primarily resistant TB bacilli.

\section{Limitations}

The data regarding pre-XDR-TB does not represent that of the entire population because the study was done among MDR-TB patients, which was not a repre- 
sentative sample of the community. Due to limitation of time and budget the treatment outcome of the pre-XDR-TB cases could not be followed up in the study.

\section{Conclusion}

This study encountered a higher rate of pre-XDR-TB among pulmonary MDR-TB patients in Bangladesh. It can be concluded that Bangladesh is in urgent need to put in action strategies for rapid and early detection of pre-XDR-TB cases among the MDR-TB patients. Identification of pre-XDR-TB cases is an important step in the prevention of treatment failure of patients suffering from MDR-TB in addition to halting the progression towards XDR-TB. Standard MDR-TB treatment regimen in pre-XDR-TB cases is of no worth rather it can amplify further resistance to the drugs that are effective. It is prudent to find out the cause for significant numbers of pre-XDR-TB cases among those with a history of regular MDR-TB treatment. If there is an ongoing high rate of transmission of primary resistant bacilli, it demands serious attention.

\section{References}

[1] NTP (2016) Annual Tuberculosis Report 2016. National Tuberculosis Control Program (NTP), Dhaka, 3. http://www.ntp.gov.bd/ntp_dashboard/magazines_image/1487245939.pdf

[2] Banerjee, R., Allen, J., Westenhouse, J., Oh, P., Elms, W., Desmond, E., Nitta, A., Royce, S. and Flood, J. (2008) Extensively Drug-Resistant Tuberculosis in California, 1993-2006. Clinical Infectious Diseases, 47, 450-457. https://doi.org/10.1086/590009

[3] Adwani, S., Desani, D.U. and Joshi, M.J. (2016) Prevalence of Pre-Extensively Drug-Resistant Tuberculosis (Pre XDR-TB) and Extensively Drug-Resistant Tuberculosis (XDR-TB) among Pulmonary Multidrug Resistant Tuberculosis (MDR-TB) at a Tertiary Care Center in Mumbai. Journal of Krishna Institute of Medical Sciences University, 5, 13-19.

[4] WHO (2016) Global Tuberculosis Report 2016, 5-49. http://www.who.int/tb/publications/global_report/en/

[5] Mannan, M.A.U., Mushtaq, A. and Naqvi, S. (2017) Prevalence of Pre-XDR-TB, XDR-TB among MDR-TB Patients Registered at PMDT Site Nishtar Hospital Multan Pakistan. European Respiratory Journal, 50, PA2764.

[6] Rao, N., Baig, S., Hussain, N., Ahmed, N. and Rao, D. (2015) Prevalence of pre-XDR-TB, XDR-TB among MDR-TB Patients Registered at Ojha Institute of Chest Diseases, Karachi. European Respiratory Journal, 46, PA2715. https://doi.org/10.1183/13993003.congress-2015.PA2715

[7] STAC (2016) Statistics \& Information of MDR and XDR TB in SAARC Region. STAC, Nepal, 23.

[8] WHO (2007) Policy Guidance on TB Drug Susceptibility Testing (DST) of Second Line Drugs (SLD). WHO Press, Geneva, Switzerland.

http://apps.who.int/iris/bitstream/handle/10665/70500/WHO_HTM_TB_2008.392_ eng.pdf?sequence $=1$

[9] Porwal, C., Kaushik, A., Makkar, N., Banavaliker, J.N., Hanif, M. and Singla, R. 
(2013) Incidences and Risk Factors for Extensively Drug Resistant Tuberculosis in Delhi Region. PLoS One, 8, e55299. https://doi.org/10.1371/journal.pone.0055299

[10] Hu, Y., Hoffner, S., Wu, L., Zhao, Q., Jiang, W. and Xua, B. (2013) Prevalence and Genetic Characterization of Second-Line Drug-Resistant and Extensively Drug-Resistant Mycobacterium tuberculosis in Rural China. Antimicrobial Agents and Chemotherapy, 57, 3857-3863. https://doi.org/10.1128/AAC.00102-13

[11] Yuan, X., Zhang, T., Kawakami, K., Zhu, J., Li, H., Lei, J. and Tu, S. (2012) Molecular Characterization of Multidrug- and Extensively Drug-Resistant Mycobacterium Tuberculosis Strains in Jiangxi, China. Journal of Clinical Microbiology, 50, 2404-2413. https://doi.org/10.1128/JCM.06860-11

[12] Singhal, P., Dixit, P., Singh, P., Jaiswal, I., Sinhg, M. and Jain, A. (2016) A Study on Pre-XDR \& XDR Tuberculosis \& Their Prevalent Genotypes in Clinical Isolates of Mycobacterium Tuberculosis in North India. The Indian Journal of Medical Research, 145, 341-347.

http://www.ijmr.org.in/article.asp?issn=0971-5916;year=2016; volume=143;issue=3; page $=341$; epage $=347$; aulast $=$ Singhal

[13] Daniel, O., Osman, E., Oladimeji, O. and Dairo, O.G. (2013) Pre-Extensive Drug Resistant Tuberculosis (Pre-XDR-TB) among MDR-TB Patents in Nigeria. Global Advanced Research Journal of Microbiology, 2, 22-25. 